\title{
Preoperatively undiagnosed papillary thyroid carcinoma in patients thyroidectomized for benign multinodular goiter
}

Fausto Fama', Alessandro Sindoni'2, Marco Cicciu', Francesca Polito ${ }^{3}$, Arnaud Piquard ${ }^{4}$, Olivier Saint-Marc ${ }^{4}$, Maria Gioffre'-Florio', Salvatore Benvenga ${ }^{3,5,6}$

\begin{abstract}
Objective: Incidental thyroid cancers (ITCs) are often microcarcinomas; among them, the most frequent histotype is the papillary one. The purpose of this study was to evaluate the rate of papillary thyroid cancer (PTC) in patients thyroidectomized for benign multinodular goiter. Subject and methods: We retrospectively evaluated the histological incidence of PTC in 207 consecutive patients who, in a 1-year period, underwent thyroidectomy for benign multinodular goiter. All patients came from an iodine-deficient area (Orleans, France) with three nuclear power stations located in the neighboring areas of the county town. Results: Overall, 25 thyroids $(12.1 \%)$ harbored 37 PTC, of which 31 were microcarcinomas. In these 25 PTC patients, mean age was $55 \pm 10$ years (range 30 75), female:male ratio $20: 5$ (4:1). In 10 patients ( $40 \%$ of 25 and $4.8 \%$ of 207 ), PTCs were bilateral, and in 7 ( 2 with microPTCs) the thyroid capsule was infiltrated. These 7 patients underwent central and lateral cervical lymph node dissections, which revealed lymph node metastases in one and two cases, respectively. Radioiodine treatment was performed in 7 cases. Neither mortality nor transient and permanent nerve injuries were observed. Four (16\%) transient hypocalcaemias occurred as early complications. At last follow-up visit (mean length of follow-up $17.2 \pm 3.4$ months), all patients were doing well and free of any clinical local recurrence or distant metastases. Conclusion: With a $12 \%$ risk that multinodular goiter harbors preoperatively unsuspected PTCs, which can have already infiltrated the capsule and that can be accompanied by PTC foci contralaterally, an adequate surgical approach has to be considered. Arch Endocrinol Metab. 2018;62(2):139-48
\end{abstract}

Keywords

Incidental thyroid cancer; benign thyroid disease; multinodular goiter; total thyroidectomy; papillary thyroid cancer
1 Department of Human Pathology in Adulthood and Childhood "G. Barresi", University Hospital of Messina, Messina, Italy

${ }^{2}$ Department of Biomedical and Dental Sciences and of Morphological and Functional Images, University Hospital of Messina, Messina, Italy ${ }^{3}$ Department of Clinical \& Experimental Medicine, University Hospital of Messina, Messina, Italy ${ }^{4}$ Department of General, Endocrine and Thoracic Surgery, Regional Hospital of Orleans, Orléans, France ${ }^{5}$ Master Program on Childhood, Adolescent and Women's Endocrine Health, University Hospital of Messina, Messina, Italy ${ }^{6}$ Interdepartmental Program on Molecular \& Clinical Endocrinology, and Women's Endocrine Health,

University Hospital of

Messina, Messina, Italy

Correspondence to: Alessandro Sindoni

Sezione di Scienze Radiologiche Dipartimento di Scienze Biomediche, Odontoiatriche e delle Immagini

Morfologiche e Funzionali,

Via Consolare Valeria, 1

98125 - Messina, Italy

alessandrosindoni@alice.it

Received on Sept/18/2016 Accepted on Oct/3/2017

DOI: $10.20945 / 2359-3997000000017$

\section{INTRODUCTION}

I $\mathrm{n}$ the thyroid literature the term incidental has been used to indicate an unsuspected finding; nevertheless, the nature of the incidental finding depends on the clinical context in which the nodules are found. Considering thyroid gland, the identification of thyroid cancer may be classified into 3 broad categories: 1) clinically detected cancer (not incidentally detected), 2) radiologically detected cancer (clinically unsuspected), and 3 ) pathologically detected cancer (clinically and radiologically unsuspected) (1). Incidental thyroid cancers (ITCs) are often microcarcinomas, most frequently of the papillary histotype (2-6); the mean tumor size of ITCs decreased during the last decades
$(3,6)$. Namely, Boucek and cols. (7) divided ITC diagnoses into four different categories: i) neoplasms found incidentally after thyroidectomy whereas preoperatively only benign pathology was known; ii) neoplasms that were diagnosed incidentally on imaging, mainly ultrasonography (US), and that were evaluated further and confirmed by fine-needle aspiration cytology (FNAC); iii) neoplasms that appeared clinically as lymph node metastases, with primary thyroid carcinoma detected only at histological specimen examination; iv) thyroid cancer that is localized in ectopic thyroid tissue with clinical symptoms or metastases present. Besides these four groups, Liu and cols. (8) proposed another ITC group including patients that presented, 
despite benign thyroid disease ascertained at imaging and definitive histology, regional or distant lymph node metastases from primary thyroid carcinoma not identified at thyroid pathological examination.

An ITC discovered at histology, after surgical removal of the thyroid for a benign pathology, is the most frequent event (9-12). In thyroidectomy specimens, ITC prevalence ranges up to $40 \%(2)$. In autopsy studies, the reported prevalence of ITC ranges from $0.01 \%$ in USA to $35.6 \%$ in Finland (7). Recently, a study from U.S.A. have documented that most counties with the highest thyroid cancer incidence are in a contiguous area of eastern Pennsylvania, New Jersey, and southern New York State; radioactive exposures from 16 nuclear power reactors within a 90-mile radius in this area have indicated that these emissions are a likely etiological factor in rising thyroid cancer incidence rates (13).

Over the last 30 years, there has been an increase in the overall incidence of thyroid cancer, from 3.6 (in 1973 ) to 8.7 (in 2002) per 100,000 inhabitants (14). The incidence rate of papillary thyroid cancer PTC rose up more than any other malignancy $(15,16)$, up to $93 \%$ of all thyroid cancers in Japan and up to $85.3 \%$ in Western countries (7). PTC is the most common histotype and microPTC represents up to $30 \%$ of all forms of papillary cancer (17).

The very recently released American Thyroid Association guidelines on thyroid nodules and cancer underscore that "a recent population based study from Olmsted County reported the doubling of thyroid cancer incidence from 2000-2012 compared to the prior decade as entirely attributable to clinically occult cancers detected incidentally on imaging or pathology" (18-20). By 2019, one study predicts that papillary thyroid cancer (PTC) will become the third most common cancer in women $(21)$.

The purpose of this study was to evaluate the rate of histologically detected PTC in consecutive patients who were thyroidectomized for benign multinodular goiter $(\mathrm{MNG})$ throughout a 1 -year period at a single endocrine surgery unit. Of note, this surgery unit and patients' residence is located near to three nuclear power units. Our data were compared with those of the English language literature on the ITCs.

\section{SUBJECTS AND METHODS}

All patients of this retrospective cohort were admitted on the same day of the surgical procedures, performed by 3 experienced endocrine surgeons under general anesthesia. Preoperatively, patients were studied by means of neck US and routine blood test, including hormones levels. The American Society of Anesthesiologists (ASA) physical status was assessed in all patients. In order to obtain a more homogeneous cohort of patients, we excluded patients with suspicious characteristics of the thyroid nodule(s) (i.e. irregular margin and/or contour and/or shape, calcifications, hypoechogenicity, vascularity or local invasion/lymph node metastases) at US $(\mathrm{n}=19)$, history of previous neck surgery $(\mathrm{n}=7)$, history of malignancy in other organs $(\mathrm{n}=5)$ and ASA score greater than $4(\mathrm{n}=2)$.

Parathyroid glands and recurrent nerves were identified in all cases, and specimens sent to pathologists for the frozen section; no cervical drains were placed systematically. Patients were discharged, generally in the second post-operative day, with a prescription of a weight-adjusted thyroxine treatment. Patients were referred to our endocrinological outpatient surveillance program. We defined microcarcinoma or macrocarcinoma any cancerous nodule up to $10 \mathrm{~mm}$ or greater than in maximum diameter, respectively. When multifocality occurred, we considered the largest neoplasm and classified according to its anatomical site. For purpose of comparison with the international literature, we run a PubMed search entering the words "incidental thyroid cancer" or "incidental thyroid carcinoma". The search was updated until November 2016. The search was limited taking into consideration only original papers. The references of the retrieved articles were also checked so as not to miss important clinical studies. Original articles reporting data about patients who underwent surgery for suspicious or preoperatively documented disease, as well as editorials, commentaries, review articles and similar types of articles were excluded. Animal studies were also excluded. Two researchers (A.S., S.B.) independently reviewed the titles and disagreements were resolved in a consensus meeting.

\section{Statistical analysis}

Results are expressed as mean \pm standard deviation (SD). Laboratory data without normal distribution were described using median and percentile values. Fisher's exact test was used to analyze categorical data. The level for statistical significance was set at $\mathrm{P}<0.05$. Statistical analysis was performed using Kyplot v2.0 beta 13 version. 


\section{RESULTS}

In our study from a French endocrine surgery unit, we retrospectively reviewed 207 consecutive patients, 169 were females (mean age of $53.0 \pm 12.6$ years [range 18-79]) and 38 males (mean age of $54.9 \pm 14.2$ years [range 21-78]), who underwent total thyroidectomy (TT) for benign bilateral MNG from January to December 2014. All patients came from an iodinedeficient area (Orleans, France) (22) with three nuclear power stations located in the neighboring areas of the county town (Figure 1). Clinico-laboratory data of all patients are shown in Table 1.

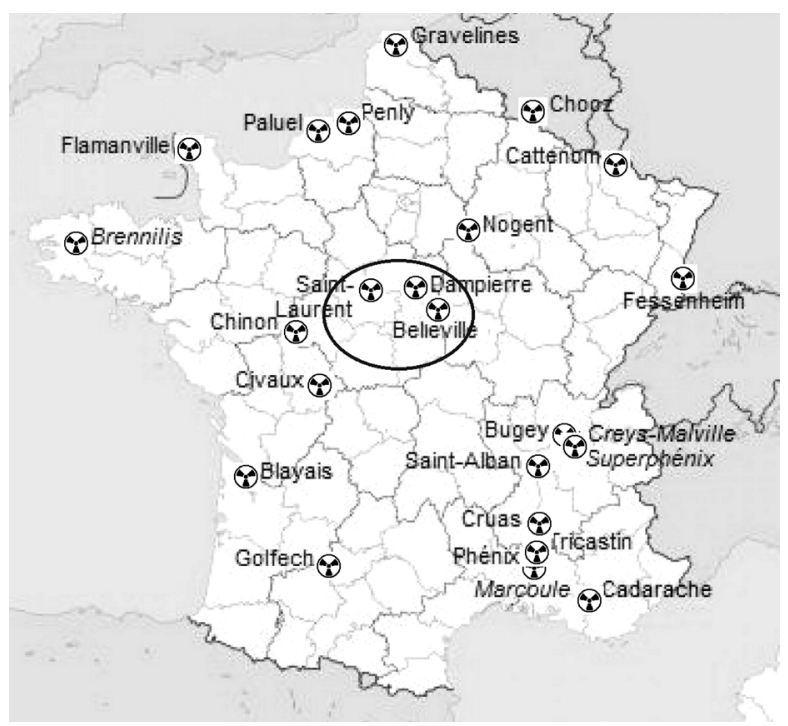

Figure 1. Topography of nuclear power plants in the neighboring areas of Orleans, France (ring).

Table 1. Demographic and clinico-laboratory characteristics of patients undergoing total thyroidectomy for benign multinodular goiter

\begin{tabular}{lc}
\hline & $\begin{array}{c}\text { All patients } \\
\text { ( } \mathbf{n}=\mathbf{2 0 7})\end{array}$ \\
\hline Age, years & \\
mean $\pm \mathrm{SD}$ & $53.9 \pm 13.9$ \\
(range) & $(18-79)$ \\
$\mathrm{TSH}, \mathrm{uUl} / \mathrm{ml}$ & \\
median & 1.54 \\
(interquartile range) & $(1.07-2.27)$ \\
$\mathrm{FT} 3$, pmol/L & \\
median & 5.6 \\
(interquartile range) & $(4.3-6.2)$ \\
FT4, pmol/L & \\
median & 15.2 \\
(interquartile range) & $(12.8-16.9)$ \\
Tg, ng/ml & \\
median & \\
(interquartile range) & 24.2 \\
\hline
\end{tabular}

SD: standard deviation; Tg: thyroglobulin.
Over the 12-month chronological window of our study, in $25 / 207$ patients (12.1\%) we discovered 37 preoperatively unsuspected, and therefore ITCs, all being PTCs. Their ASA score of these patients was ASAl $(\mathrm{n}=4)$, ASA2 $(\mathrm{n}=18)$ and ASA3 $(\mathrm{n}=3)$. Mean hospital stay was $1.1 \pm 0.3$ days; $23(92 \%)$ were discharged on the $1^{\text {st }}$ post-operative day and 2 on the $2^{\text {nd }}$ post-operative day.

Of these 37 PTCs, 31 (86.1\%) were microPTCs, with a maximum diameter ranging $\mathrm{l}$ to $6 \mathrm{~mm}$, while 6 were macroPTCs (diameter range $12-16 \mathrm{~mm}$ ). Overall, mean age of the 25 patients was $55 \pm 10$ years (range 30-75) with 20 being females (F:M ratio $=$ 4:1). Patients with macroPTCs were 7 years older than patients with microPTCs (Table 2). Histopathological examination showed bilateral MNG in all cases (mean weight of the thyroid glands: $53.6 \pm 45.7 \mathrm{~g}$ ) and the additional presence of chronic lymphocytic thyroiditis or Hashimoto's thyroiditis (HT) in 6/25 patients (24\%, all with positive thyroid peroxidase and thyroglobulin autoantibodies). Thyroid tumors were monofocal in 15 patients (all microPTCs; 15/37 tumors in 15 patients) and multifocal in 10. Of these multifocal PTCs, 16 were microcarcinomas and 6 macrocarciomas. In 5 of the 10 patients microPTCs and macroPTC coexisted.

Multifocal PTCs, including coexistence of micro ( $\mathrm{n}$ $=16$ ) and macroPTCs $(\mathrm{n}=6)$, were always bilateral. Of the 15 monofocal microPTCs, 8 were right-sided, and 7 left-sided (Table 1). MicroPTCs and macroPTCs did not differ in distribution if we considered the right lobe-left lobe-isthmus location ( $\mathrm{P}=0.836$ by Fisher's exact test) or the classification among the uppermiddle-lower-isthmic localization in the thyroid ( $\mathrm{P}=$ 0.334 by Fisher's exact test). Of the $6 / 207$ patients with HT, $2 / 6(33.3 \%)$ had 4 of the 37 PTCs, all 4 tumors being microPTCs.

Seven supplementary central and lateral cervical lymph node dissections were carried out, because 2 microPTCs and 5 PTCs were infiltrating the thyroid capsule at frozen sections. Lymph node metastases were found in one and two patients, respectively. Radioiodine treatment, with a dose of $100 \mathrm{mCi}$, was performed in 7 cases, because of the presence of poor prognostic factors such as capsular infiltration, macroPTC and/or multifocality.

Neither mortality nor transient and permanent nerve injuries were observed. Four (16\%) transient hypocalcaemias occurred as early complications, and were successfully treated by a 6 -week combined cholecalciferol and oral calcium supplementation. 
Table 2. PTC patients and tumours characteristics

\begin{tabular}{|c|c|c|c|c|}
\hline & Right lobe & Left lobe & Isthmus & Total \\
\hline No. of nodules & 17 & 16 & 4 & 37 \\
\hline$(\%)$ & $(46.0 \%)$ & $(43.2 \%)$ & $(10.8 \%)$ & $(100 \%)$ \\
\hline No. of patients with multifocality & 4 & 5 & 1 & $10 / 25$ \\
\hline (\%) & $(16 \%)$ & $(20 \%)$ & $(4 \%)$ & $(40.0 \%)$ \\
\hline \multicolumn{5}{|l|}{ microPTC } \\
\hline No. of nodules & 14 & 14 & 3 & 31 \\
\hline$(\%)$ & $(45.2 \%)$ & $(45.2 \%)$ & $(9.6 \%)$ & \\
\hline Mean diameter $(\mathrm{mm}) \pm \mathrm{SD}$ & $4.4 \pm 2.7$ & $3.9 \pm 2.5$ & 1 & $3.8 \pm 2.6$ \\
\hline Mean age (years) $\pm S D$ & $55.9 \pm 10.2$ & $52.2 \pm 7.7$ & $57.0 \pm 18.7$ & $54.4 \pm 9.9$ \\
\hline F:M ratio & $3.7: 1$ & $3.7: 1$ & $3: 0$ & $4.2: 1$ \\
\hline \multicolumn{5}{|l|}{ macroPTC } \\
\hline No. of nodules & 3 & 2 & 1 & 6 \\
\hline$(\%)$ & $(50 \%)$ & $(33.3 \%)$ & $(16.7 \%)$ & \\
\hline Mean diameter $(\mathrm{mm}) \pm \mathrm{SD}$ & $13.0 \pm 1.7$ & $12.5 \pm 0.7$ & 30 & $15.7 \pm 7.1$ \\
\hline Mean age (years) \pm SD & $60.0 \pm 13.5$ & $67.5 \pm 9.2$ & 52 & $61.2 \pm 11.1$ \\
\hline F:M ratio & 3:0 & $2: 0$ & $0: 1$ & $5: 1$ \\
\hline
\end{tabular}

PTC: papillary thyroid carcinoma; SD: standard deviation; F: female; M, male.

At last follow-up visit (mean length of follow-up $17.2 \pm 3.4$ months), all patients were doing well and free of any clinical local recurrence or distant metastases.

An overview of the literature is summarized in Table 3 (23-68). Reported prevalence of ITC at surgery ranges between $2 \%$ and $40 \%(1,2,17,23-68)$ : in Europe it varies from $2.2 \%$ to $27.4 \%$ and in the United States it varies from $3.3 \%$ to $33 \%$. In some European countries, such as Romania, Czech Republic, Ukraine and Poland, the frequency of thyroid cancer showed a lower range (i.e. from 5 to $9.2 \%$ ); in Turkey, excluding the study from Tasova and cols. (46), there has been a lower variation range in its reported incidence (7-10\%). Rates from other European countries were: $12.5 \%$ from Belgium, 10.4-11.1\% from Italy and 12.0\% from Greece.

Table 3. Summary of the literature on thyroid cancers that were discovered incidentally at thyroidectomy in patients underwent surgery for benign thyroid disease

\begin{tabular}{|c|c|c|c|c|c|c|}
\hline Author (ref) & $\begin{array}{l}\text { Years of } \\
\text { study }\end{array}$ & Country & $\begin{array}{l}\text { Patients } \\
\text { studied }\end{array}$ & Surgical procedure & Rate of cancer & Comment \\
\hline $\begin{array}{l}\text { Fama' and cols., this } \\
\text { study }\end{array}$ & One (2014) & France & $207 \mathrm{pts}$ & TT & $\begin{array}{l}\text { In 25/207 (12.1\%) pts, } 37 \\
\text { PTC were detected ( } 31 \\
\text { microPTCs and } 6 \text { PTCs) }\end{array}$ & $\begin{array}{c}10 / 25(40.0 \%) \text { pts had } \\
\text { multifocal tumours; all pts } \\
\text { underwent surgery for MNG }\end{array}$ \\
\hline $\begin{array}{l}\text { Daumerie and cols., } \\
1998\end{array}$ & $1976-1995$ & Belgique & $93 \mathrm{pts}$ & $\begin{array}{c}\text { TT in 16/47 (34.0\%) pts } \\
\text { with MNG (group I), PT in } \\
39 / 46 \text { (84.8\%) pts with a } \\
\text { solitary hot nodule (group II) }\end{array}$ & $\begin{array}{l}\text { 2/16 (12.5\%) pts (group I) } \\
\text { and } 5 / 39(12.8 \%) \text { pts (group } \\
\text { II) had microTC, with a total } \\
\text { prevalence of } 12.7 \%\end{array}$ & \\
\hline $\begin{array}{l}\text { Dănilă and cols., } \\
2008\end{array}$ & $2000-2006$ & Germany & $92 \mathrm{pts}$ & $\pi$ & $\begin{array}{l}2 / 92(2.2 \%) \text { pts had } \\
\text { microPTC (tumour size } \\
\text { ranged from } 3 \text { to } 5 \mathrm{~mm} \text { ) }\end{array}$ & $\begin{array}{l}\text { All pts had GD; multifocality } \\
\text { and lymph node involvement } \\
\text { were not detected }\end{array}$ \\
\hline $\begin{array}{l}\text { Pezzolla and cols., } \\
2014\end{array}$ & $\begin{array}{l}\text { Jan } 2010 \\
\text { - Jun } 2013\end{array}$ & Italy & $256 \mathrm{pts}$ & $\begin{array}{l}\text { Surgical procedures in } \\
\text { tumours pts 28/256): TT in } \\
27 / 28, \text { PT in 1/28 PT }\end{array}$ & $\begin{array}{l}\text { In } 28 / 256(10.9 \%) \text { pts, } 40 \\
\text { TCs were detected (29 } \\
\text { FV-PTC, } 10 \text { PTC and } 1 \text { FTC) }\end{array}$ & $\begin{array}{l}\text { Pts underwent surgery for: } \\
176 \text { pts (MNG), } 67 \text { pts (GD), } \\
12 \text { pts (UNG) and } 1 \text { pt (PD) }\end{array}$ \\
\hline $\begin{array}{l}\text { Pezzolla and cols., } \\
2010\end{array}$ & $\mathrm{n} / \mathrm{a}$ & Italy & $165 \mathrm{pts}$ & $\mathrm{n} / \mathrm{a}$ & $\begin{array}{c}\text { 30/165 (18.2\%) pts had TC } \\
\text { (18 PTC, } 6 \text { FTC, } 5 \text { FV-PTC } \\
\text { and } 1 \text { oncocytic carcinoma); } \\
\text { 15/30 (50\%) were } \\
\text { microcarcinomas }\end{array}$ & $\begin{array}{l}\text { Pts underwent surgery for: } \\
132 \text { pts (MNG), } 30 \text { pts } \\
\text { (UNG), } 2 \text { pts (PD) and } 1 \text { pt } \\
\text { (GD) }\end{array}$ \\
\hline
\end{tabular}




\begin{tabular}{|c|c|c|c|c|c|c|}
\hline Author (ref) & $\begin{array}{l}\text { Years of } \\
\text { study }\end{array}$ & Country & $\begin{array}{l}\text { Patients } \\
\text { studied }\end{array}$ & Surgical procedure & Rate of cancer & Comment \\
\hline $\begin{array}{l}\text { Costamagna and } \\
\text { cols., } 2013\end{array}$ & $2001-2009$ & Italy & $568 \mathrm{pts}$ & $\begin{array}{c}\text { TT in 499/568, PT in } \\
69 / 568\end{array}$ & $\begin{array}{c}\text { 53/568 (9.3\%) pts had TC } \\
\text { (24 FV-PTC, } 20 \text { PTC, } 4 \text { FTC, } \\
4 \text { MTC and } 1 \text { primitive } \\
\text { thyroid paraganglioma); } \\
\text { 32/53 (60.4\%) had } \\
\text { microPTCs }\end{array}$ & $\begin{array}{c}14 / 53(26.4 \%) \text { pts had } \\
\text { multifocal tumours and in } \\
12 / 53(22.6 \%) \text { were bilateral }\end{array}$ \\
\hline $\begin{array}{l}\text { Negro and cols., } \\
2013^{\star}\end{array}$ & $2000-2010$ & Italy & $970 \mathrm{pts}$ & $\pi$ & 84/ 970 (8.7\%) pts had TC & \\
\hline $\begin{array}{l}\text { Botrugno and cols., } \\
2011\end{array}$ & $2000-2008$ & Italy & $462 \mathrm{pts}$ & $\pi$ & $\begin{array}{c}\text { 41/462 (8.9\%) pts had TC; } \\
\text { the most common histotype } \\
\text { was PTC }\end{array}$ & \\
\hline $\begin{array}{l}\text { Gelmini and cols., } \\
2010\end{array}$ & $10 \mathrm{yrs}$ & Italy & $739 \mathrm{pts}$ & $\begin{array}{c}\text { TT in 503/739, PT in } \\
\text { 239/739 }\end{array}$ & $\begin{array}{c}82 / 739(11.1 \%) \text { pts had TC, } \\
\text { mainly microPTC }\end{array}$ & $\begin{array}{l}\text { Lymph-node metastases } \\
\text { were found in the } 3.6 \% \text { of } \\
\text { cases }\end{array}$ \\
\hline $\begin{array}{l}\text { Pisello and cols., } \\
2007\end{array}$ & $\begin{array}{l}\text { Jan } 2000 \\
- \text { Jan } 2006\end{array}$ & Italy & $502 \mathrm{pts}$ & $\begin{array}{c}\text { TT in 458/502, PT in } \\
44 / 502\end{array}$ & $\begin{array}{c}\text { 17/502 (3.4\%) pts had } \\
\text { microPTC }\end{array}$ & $\begin{array}{c}\text { In } 34 / 502(6.8 \%) \text { pts, } \\
\text { tumours were suspected } \\
\text { preoperatively; } 2 / 502(0.4 \%) \\
\text { had multifocal microPTCs }\end{array}$ \\
\hline $\begin{array}{l}\text { Carlini and cols., } \\
2006^{\star}\end{array}$ & $\mathrm{n} / \mathrm{a}$ & Italy & $88 \mathrm{pts}$ & $\pi$ & 19/88 (21.6\%) pts had TC & \\
\hline $\begin{array}{l}\text { Miccoli and cols., } \\
2006\end{array}$ & $\begin{array}{l}\text { Feb } 2002 \\
\text { - Nov } 2003\end{array}$ & Italy & 998 pts & $\begin{array}{l}\text { TT in 902/998 pts, PT in } \\
\text { 96/998 }\end{array}$ & $\begin{array}{l}\text { 104/998 (10.4\%) pts had } \\
\text { TC; the most common } \\
\text { histotype was PTC (99/104) }\end{array}$ & $\begin{array}{c}\text { Tumours were multifocal in } \\
19.8 \% \text { of the cases }\end{array}$ \\
\hline $\begin{array}{l}\text { Carlini and cols., } \\
2005^{\star}\end{array}$ & 1-year & Italy & $\mathrm{n} / \mathrm{a}$ & $\mathrm{n} / \mathrm{a}$ & $\begin{array}{c}\text { Incidence of microTC was } \\
27.4 \%\end{array}$ & \\
\hline $\begin{array}{l}\text { Pingitore and cols., } \\
1993\end{array}$ & $1985-1991$ & Italy & $2930 \mathrm{pts}$ & $\mathrm{n} / \mathrm{a}$ & 132/2463 (5.4\%) pts had TC & $\begin{array}{l}\text { 2463/2930 pts were } \\
\text { considered clinically benign } \\
\text { and } 467 / 2930 \text { pts } \\
\text { malignant, preoperatively }\end{array}$ \\
\hline $\begin{array}{l}\text { Pascual Corrales } \\
\text { and cols., } 2012\end{array}$ & $\mathrm{n} / \mathrm{a}$ & Spain & 372 pts & $\mathrm{n} / \mathrm{a}$ & 58/372 (15.6\%) pts had TC & $\begin{array}{l}\text { Pts underwent surgery for: } \\
49 \text { pts (EMNG), } 8 \text { pts (GD) } \\
\text { and } 1 \text { pt (HMNG) }\end{array}$ \\
\hline $\begin{array}{l}\text { Slijepcevic and cols., } \\
2015\end{array}$ & $2008-2013$ & Serbia & $2466 \mathrm{pts}$ & TT or PT & $\begin{array}{c}\text { 403/2466 (16.3\%) pts had } \\
\text { microPTC }\end{array}$ & \\
\hline $\begin{array}{l}\text { Zivaljević and cols., } \\
2008\end{array}$ & 2004 & Serbia & $578 \mathrm{pts}$ & $\mathrm{n} / \mathrm{a}$ & $\begin{array}{c}53 / 578 \text { (9.2\%) pts had } \\
\text { microTC }\end{array}$ & $\begin{array}{l}\text { Pts underwent surgery for: } \\
201 \text { pts (MNG), } 178 \text { pts } \\
\text { (thyroid adenoma), } 89 \text { pts } \\
\text { (GD), } 79 \text { pts (PD) and } 31 \text { pts } \\
\text { thyroiditis }\end{array}$ \\
\hline $\begin{array}{l}\text { Alecu and cols., } \\
2014\end{array}$ & $2002-2012$ & Romania & $145 \mathrm{pts}$ & $\begin{array}{c}\text { TT in } 102 / 145 \text { pts, PT in } \\
43 / 145\end{array}$ & $\begin{array}{c}\text { 10/145 (6.9\%) pts had } \\
\text { microPTC }\end{array}$ & \\
\hline $\begin{array}{l}\text { Muntean and cols., } \\
2013\end{array}$ & $2002-2011$ & Romania & $2168 \mathrm{pts}$ & TT or PT & $\begin{array}{l}\text { 187/2168 (8.6\%) pts had } \\
\text { microPTC }\end{array}$ & $\begin{array}{c}\text { In } 66 / 187(35.3 \%) \text { pts had } \\
\text { multifocal tumours, and in } \\
31 / 187(16.6 \%) \text { were } \\
\text { bilateral }\end{array}$ \\
\hline $\begin{array}{l}\text { Lukás and cols., } \\
2010\end{array}$ & $2004-2008$ & $\begin{array}{c}\text { Czech } \\
\text { Republic }\end{array}$ & $400 \mathrm{pts}$ & TT or PT & $\begin{array}{c}\text { 34/400 (8.5\%) pts had } \\
\text { microTC; } 32 / 34 \text { (94.1\%) } \\
\text { were microPTCs }\end{array}$ & $\begin{array}{c}\text { In 5/34 (14.7\%) pts had } \\
\text { multifocal tumours and in } \\
\text { 4/34 (11.8\%) were bilateral }\end{array}$ \\
\hline $\begin{array}{l}\text { Nechă and cols., } \\
2012^{\star}\end{array}$ & $2008-2009$ & Ukraine & $608 \mathrm{pts}$ & $\mathrm{n} / \mathrm{a}$ & $\begin{array}{c}\text { 56/608 (9.2\%) pts had TC; } \\
\text { 43/56 (76.8\%) were } \\
\text { microPTCs }\end{array}$ & \\
\hline $\begin{array}{l}\text { Barczyński and } \\
\text { cols., 2011* }\end{array}$ & $1999-2009$ & Poland & $8132 \mathrm{pts}$ & $\begin{array}{c}\text { TT in 2918/8132 pts, PT in } \\
5214 / 8132\end{array}$ & 406/8132 (5.0\%) pts had TC & \\
\hline $\begin{array}{l}\text { Vasileiadis and cols., } \\
2013\end{array}$ & $2001-2009$ & Greece & $2236 \mathrm{pts}$ & $\pi$ & $\begin{array}{c}\text { 268/2236 (12.0\%) pts had } \\
\text { microPTC. }\end{array}$ & \\
\hline
\end{tabular}




\begin{tabular}{|c|c|c|c|c|c|c|}
\hline Author (ref) & $\begin{array}{l}\text { Years of } \\
\text { study }\end{array}$ & Country & $\begin{array}{l}\text { Patients } \\
\text { studied }\end{array}$ & Surgical procedure & Rate of cancer & Comment \\
\hline $\begin{array}{l}\text { Siassakos and cols., } \\
2008\end{array}$ & $\begin{array}{c}\text { Jan } 1997 \text { - Jul } \\
2001\end{array}$ & Greece & 191 pts & $\pi$ & $\begin{array}{l}\text { 29/191 (15.2\%) pts had } \\
\text { microTC (18 microFTC, } 10 \\
\text { microPTC and } 1 \text { microMTC) }\end{array}$ & $\begin{array}{c}\text { In } 8 / 29(27.6 \%) \text { pts had } \\
\text { multifocal microcarcinomas }\end{array}$ \\
\hline $\begin{array}{l}\text { Sakorafas and cols., } \\
2007\end{array}$ & $\begin{array}{l}\text { Feb } 1990 \\
\text { - Feb } 2002\end{array}$ & Greece & $380 \mathrm{pts}$ & $\begin{array}{c}\text { TT in } 377 / 380 \text { pts, } P \text { T in } \\
3 / 380\end{array}$ & $\begin{array}{c}27 / 380(7.1 \%) \text { pts had } \\
\text { microPTC }\end{array}$ & $\begin{array}{l}\text { In 11/27 (40.7\%) pts had } \\
\text { multifocal tumours }\end{array}$ \\
\hline $\begin{array}{l}\text { Yazici and cols., } \\
2015\end{array}$ & $2010-2013$ & Turkey & $86 \mathrm{pts}$ & TT or PT & $\begin{array}{c}\text { 6/86 (7.0\%) pts had TC (4 } \\
\text { microPTC and } 2 \text { PTC) }\end{array}$ & \\
\hline $\begin{array}{l}\text { Tasova and cols., } \\
2013\end{array}$ & $\begin{array}{l}\text { Mar } 2007 \\
\text { - May } 2011\end{array}$ & Turkey & $443 \mathrm{pts}$ & $\begin{array}{c}\text { TT in 401/443, PT in } \\
42 / 443\end{array}$ & $\begin{array}{l}\text { 66/443 (14.9\%) pts had TC } \\
\text { (56 PTC, } 4 \text { FTC and } 6 \\
\text { indeterminate lesions) }\end{array}$ & \\
\hline $\begin{array}{l}\text { Karakoyun and cols., } \\
2013\end{array}$ & $\begin{array}{l}\text { Jan } 2010 \\
\text { - Aug } 2011\end{array}$ & Turkey & $50 \mathrm{pts}$ & TT & $\begin{array}{l}5 / 50(10.0 \%) \text { pts had } \\
\text { microPTC. }\end{array}$ & \\
\hline $\begin{array}{l}\text { Berker and cols., } \\
2011\end{array}$ & $\begin{array}{l}\text { Jan } 2004 \\
\text { - Jan } 2009\end{array}$ & Turkey & $337 \mathrm{pts}$ & $\pi$ & $\begin{array}{c}\text { 18/337 (5.3\%) pts had } \\
\text { microTC }\end{array}$ & $\begin{array}{l}\text { Pts underwent surgery for: } \\
278 \text { pts (MNG), } 59 \text { pts (GD) }\end{array}$ \\
\hline $\begin{array}{l}\text { Tezelman and cols., } \\
2009\end{array}$ & $1988-2007$ & Turkey & $2906 \mathrm{pts}$ & $\begin{array}{l}\text { PT in 1695/2906 (group 1), } \\
\text { TT in 1211/2906 (group 2) }\end{array}$ & $\begin{array}{c}\text { 210/2906 (7.2\%) pts had TC } \\
\text { (81 in group } 1 \text { and } 129 \text { in } \\
\text { group 2) }\end{array}$ & \\
\hline $\begin{array}{l}\text { Giles and cols., } \\
2004\end{array}$ & $\begin{array}{l}\text { Sep } 2001 \\
\text { - Dec } 2002\end{array}$ & Turkey & 218 pts & $\begin{array}{c}\Pi \text { in 109/218 (group 1), } \\
\text { and PT in 109/218 (group 2) }\end{array}$ & $\begin{array}{c}\text { 18/218 (8.3\%) pts had PTC } \\
\text { (10 in group } 1 \text { and } 8 \text { in } \\
\text { group 2) }\end{array}$ & $\begin{array}{l}\text { All pts underwent surgery for } \\
\text { MNG }\end{array}$ \\
\hline $\begin{array}{l}\text { Fernando and cols., } \\
2009\end{array}$ & $2003-2005$ & Sri Lanka & $68 \mathrm{pts}$ & $\pi$ & $\begin{array}{l}\text { 6/68 (8.8\%) pts had TC (2 } \\
\text { PTC, } 2 \text { MTC and } 2 \text { FTC) }\end{array}$ & \\
\hline $\begin{array}{l}\text { John and cols., } \\
2014\end{array}$ & $\begin{array}{l}\text { Jan } 2005 \\
\text { - Jun } 2012\end{array}$ & India & $1300 \mathrm{pts}$ & TT or PT & $\begin{array}{c}94 / 1300(7.2 \%) \text { pts had } \\
\text { microPTC }\end{array}$ & \\
\hline Wu and cols., 1993* & $1962-1991$ & China & $135 \mathrm{pts}$ & $n / a$ & $54 / 135$ (40.0\%) pts had TC & \\
\hline Koh and cols., 1992 & $\mathrm{n} / \mathrm{a}$ & Malaysia & 107 pts & $\mathrm{n} / \mathrm{a}$ & $\begin{array}{c}\text { 8/107 (7.5\%) pts had TC, } \\
\text { mainly PTC }\end{array}$ & $\begin{array}{l}\text { All pts underwent surgery for } \\
\text { MNG }\end{array}$ \\
\hline $\begin{array}{l}\text { Preece and cols., } \\
2014\end{array}$ & $\begin{array}{l}\text { Sep } 1994 \\
\text { - Aug } 2012\end{array}$ & Australia & $1508 \mathrm{pts}$ & $\pi$ & 96/1508 (6.4\%) pts had TC & $\begin{array}{l}\text { Pts underwent surgery for: } \\
963 \text { pts (MNG), } 295 \text { pts } \\
\text { (TNG), } 250 \text { pts (GD) }\end{array}$ \\
\hline Bron and cols., 2004 & 1998-2002 & Australia & 834 pts & $\pi$ & $\begin{array}{c}\text { 71/834 (8.5\%) pts had TC } \\
\text { (33 microPTC, } 22 \text { PTC, } 11 \\
\text { FTC, } 5 \text { other) }\end{array}$ & $\begin{array}{c}\text { 74/834 had previously } \\
\text { undergone PT }\end{array}$ \\
\hline $\begin{array}{l}\text { Bhuiyan and cols., } \\
2015\end{array}$ & $2003-2008$ & South Africa & $90 \mathrm{pts}$ & TT & $\begin{array}{c}10 / 90(11.1 \%) \text { pts had TC (3 } \\
\text { PTC and } 7 \text { FTC) }\end{array}$ & \\
\hline $\begin{array}{l}\text { Bombil and cols., } \\
2014\end{array}$ & $2005-2010$ & South Africa & 162 pts & $\pi$ & $\begin{array}{c}\text { 4/162 (2.5\%) pts had PTC } \\
\text { (3/4 were FV-PTC) }\end{array}$ & \\
\hline $\begin{array}{l}\text { Edino and cols., } \\
2010\end{array}$ & $2000-2006$ & Nigeria & 160 pts & $\mathrm{n} / \mathrm{a}$ & $\begin{array}{c}\text { 24/160 (15.0\%) pts had TC } \\
\text { (13 FTC, } 10 \text { PTC, } 1 \text { MTC and } \\
1 \text { ATC) }\end{array}$ & $\begin{array}{c}\text { In } 6 / 25(24.0 \%) \text { pts tumours } \\
\text { were detected preoperatively } \\
\text { by FNAC }\end{array}$ \\
\hline $\begin{array}{l}\text { Choong and cols., } \\
2015\end{array}$ & $1990-2014$ & USA & 148 pts & TT (120 pts) or PT (28 pts) & $\begin{array}{l}\text { 7/148 (4.7\%) pts had TC ( } 5 \\
\text { PTC, } 1 \text { FTC and } 1 \text { MTC) }\end{array}$ & $\begin{array}{c}\text { All pts underwent surgery for } \\
\text { TNG }\end{array}$ \\
\hline $\begin{array}{l}\text { Ergin and cols., } \\
2014\end{array}$ & $2005-2013$ & USA & 493 pts & $\pi$ & $\begin{array}{l}69 / 248(28 \%) \text { pts in EG } \\
\text { group and } 64 / 245(26 \%) \text { pts } \\
\text { in GD group had microPTC }\end{array}$ & $\begin{array}{l}\text { Pts underwent surgery for: } \\
248 \text { pts (EG), } 245 \text { pts (GD) }\end{array}$ \\
\hline $\begin{array}{l}\text { Bahl and cols., } \\
2014^{*}\end{array}$ & 2003-2012 & USA & $2090 \mathrm{pts}$ & $\mathrm{n} / \mathrm{a}$ & 680/2090 (33\%) pts had TC & \\
\hline $\begin{array}{l}\text { Phitayakorn and } \\
\text { cols., } 2013\end{array}$ & $\begin{array}{l}\text { Dec } 1985 \\
\text { - Mar } 2010\end{array}$ & USA & 300 pts & TT or PT & $\begin{array}{c}\text { 31/300 (10.3\%) pts had TC } \\
\text { (22 microPTC, } 8 \text { PTC and } 1 \\
\text { FTC) }\end{array}$ & \\
\hline $\begin{array}{l}\text { Phitayakorn and } \\
\text { cols., } 2008\end{array}$ & 1990-2007 & USA & $506 \mathrm{pts}$ & $\Pi$, PT in 10 pts with GD & $\begin{array}{c}\text { 11/333 (3.3\%) nonTNG pts } \\
\text { had PTC, 2/92 (2.2\%) GD } \\
\text { pts had microPTC, } 5 / 81 \\
\text { (6.2\%) TNG pts had TC ( } 3 \\
\text { PTC, } 1 \text { FTC and 1 MTC) }\end{array}$ & $\begin{array}{l}\text { Pts underwent surgery for: } \\
333 \text { pts (nonTNG), } 92 \text { pts } \\
\text { (GD), } 81 \text { pts (TNG) }\end{array}$ \\
\hline
\end{tabular}




\begin{tabular}{|c|c|c|c|c|c|c|}
\hline Author (ref) & $\begin{array}{l}\text { Years of } \\
\text { study }\end{array}$ & Country & $\begin{array}{l}\text { Patients } \\
\text { studied }\end{array}$ & Surgical procedure & Rate of cancer & Comment \\
\hline $\begin{array}{l}\text { Smith and cols., } \\
2013\end{array}$ & $2000-2011$ & USA & 1523 pts & $\mathrm{n} / \mathrm{a}$ & $\begin{array}{c}\text { 238/1523 (15.6\%) pts had } \\
\text { TC (175 PTC, } 39 \text { FV-PTC, } 11 \\
\text { FTC and } 13 \text { other } \\
\text { malignancies) }\end{array}$ & \\
\hline $\begin{array}{l}\text { Smith and cols., } \\
2013^{\star}\end{array}$ & 2002-2011 & USA & 164 pts & $\mathrm{n} / \mathrm{a}$ & 30/164 (18.3\%) pts had TC & $\begin{array}{c}\text { All pts underwent surgery for } \\
\text { TNG }\end{array}$ \\
\hline $\begin{array}{l}\text { Dunki-Jacobs and } \\
\text { cols., } 2012\end{array}$ & $2001-2007$ & USA & 723 pts & TT or PT & $\begin{array}{l}\text { 194/723 (27\%) pts had TC } \\
\text { (PTC or microPTC) }\end{array}$ & $\begin{array}{l}\text { In 137/194 (70.6\%) pts, } \\
\text { tumours were suspected } \\
\text { preoperatively }\end{array}$ \\
\hline $\begin{array}{l}\text { Bradly and cols., } \\
2009\end{array}$ & $\begin{array}{l}\text { Jan } 2000 \\
\text { - May } 2008\end{array}$ & USA & $678 \mathrm{pts}$ & TT or PT & $81 / 678$ (12\%) pts had PTC & \\
\hline $\begin{array}{l}\text { Lokey and cols., } \\
2005^{\star}\end{array}$ & $\begin{array}{l}\text { Dec } 1998 \\
\text {-Dec } 2003\end{array}$ & USA & $738 \mathrm{pts}$ & $\mathrm{n} / \mathrm{a}$ & $\begin{array}{l}\text { 28/738 (3.8\%) pts had TC } \\
\text { (mainly microPTC) }\end{array}$ & \\
\hline
\end{tabular}

pts: patients; TT: total thyroidectomy; PT: partial thyroidectomy; TC: thyroid carcinoma; PTC: papillary thyroid carcinoma; FV-PTC: follicular variant of papillary thyroid carcinoma; FTC: follicular thyroid carcinoma; MTC: medullary thyroid carcinoma; ATC: anaplastic thyroid carcinoma; MNG: multinodular goiter; UNG: uninodular goiter; TNG, toxic nodular goiter; GD, Graves' disease; PD, Plummer's disease; HT, Hashimoto's thyroiditis; EG, euthyroid goiter; EMNG, euthyroid multinodular goiter; HMNG, hyperthyroid multinodular goiter, FNAC: fine-needle aspiration cytology; n/a: not available.

* Tabulated data taken from the abstracts written in English and/or illustrative material.

\section{DISCUSSION}

The increased incidence of thyroid carcinoma seems to be related to an improved diagnostic approach, given by a widespread use of US and cytology, but also by the employment of new imaging techniques, such as ${ }^{18} \mathrm{~F}$-fluoro-deoxyglucose positron emission tomogram/computed tomography ( ${ }^{18} \mathrm{~F}-\mathrm{FDG}-\mathrm{PET} /$ CT) (69-71). Among patients who performed neck US for suspected parathyroid disease, incidental thyroid nodules were found in $46 \%$ of them (72). Similarly, thyroid incidentalomas discovered during CT or magnetic resonance imaging that had been carried out for other reasons have been reported with an incidence of $16 \%(73,74)$; moreover, $9 \%$ to $13 \%$ were discovered during carotid US $(75,76)$, and $2 \%$ to $3 \%$ at ${ }^{18} \mathrm{~F}-\mathrm{FDG}$ PET/CT scan (77-79). The prevalence of incidental thyroid nodules on US in the general population ranges between $42 \%$ and $67 \%(80,81)$. In thyroidectomy specimens, ITC prevalence ranges up to $40 \%$ (2). In autopsy studies, the reported prevalence of ITC ranges from $0.01 \%$ in USA to $35.6 \%$ in Finland (7).

The overview of the literature (Table 3 , refs. 23$68)$, has shown that one-third $(n=16)$ of the studies are on cohorts of thyroidectomized patients smaller than ours ( $\mathrm{n}=50$ to 191 , compared to 207 ), and oneseventh of the studies $(\mathrm{n}=7)$ are on cohorts slightly greater than ours $(256$ to 8,132$)$. Prevalence of ITC at surgery ranges between $2 \%$ and $40 \%(1,2,17,23$ $68)$. In Europe, the frequency of ITC varies from $2.2 \%$ to $27.4 \%$, and a similar wide range $(3.3 \%$ to $33 \%)$ is observed in the United States. Interestingly, in Eastern Europe (Romania, Czech Republic, Ukraine, Poland), the frequency of thyroid cancer is relatively low (range 5-9.2\%). In Turkey, excluding the study from Tasova and cols. (46), there is a lower variation range in the reported incidence of thyroid cancer (7-10\%).

One comment deserves the coexistence of ITCs with HT. We found a $33 \%$ rate of ITCs (always microPTCs) in patients with histologically confirmed HT. This rate is greater than that reported in one recent retrospective study from Serbia (37). Slijepcevic and cols. (37) also investigated the prevalence of microPTC in patients operated for benign thyroid diseases in a retrospective study of 2,466 patients who underwent thyroid surgery from 2008 to 2013 . The overall prevalence of microPTC was $16.3 \%$, the highest being in HT. Smith and cols. (63) examined cancer frequency in patients referred for removal of benign thyroid disease in a multiinstitutional series of 2,551 patients. Indeterminate/ malignant FNA diagnoses were excluded $(n=1,028)$. Overall, $238(15.6 \%)$ cancers were found, and 275 patients had thyroiditis (18\%). Presence of thyroiditis was not associated with cancer, because there were 47 ITCs in the 275 patients compared with 191 ITCs in 1,247 patients without thyroiditis ( $17.1 \%$ vs $15.3 \%$ ). Our rate of $33.3 \%$ was highly significant as well as the $22.7 \%\left(\chi^{2}=10.80, \mathrm{P}<0.001\right)$ of Slijepcevic and cols. (37), whereas the rate of $17.1 \%\left(\chi^{2}=0.388, P=0.533\right)$ reported by Smith and cols. did not reach statistical significance. 
The limitations of this study are due to its retrospective nature. Another limitation is the natural history of thyroid cancer, which is a slow growing tumor, so that extended follow-up is needed to evaluate the long-term outcomes. The strength of the study lies in its short course, avoiding that a variable number of pathologists histologically examined the specimens using different methods of evaluation.

Our $12.1 \%$ rate is comparable to rates from other European countries, including Belgium, Italy $(25,30,33)$ and Greece (17). Because Italy and Greece have no nuclear plants, we tend to exclude that our rate was influenced by the relative vicinity of our medical center and residence of patients to three nuclear plant units (82). A systematic review and meta-analysis on this issue does not support an association between living near nuclear power plants and risk of thyroid cancer. However, sensitivity analysis by exposure definition demonstrated that living less than $20 \mathrm{~km}$ from nuclear power plants was associated with a significant increase in the risk of thyroid cancer (83). Additionally, with a $12 \%$ risk that MNG harbors preoperatively unsuspected PTCs which can have already infiltrated the capsule and that are accompanied frequently by other PTC foci contralaterally, an adequate surgical approach has to be considered.

The operative management of benign thyroid diseases includes partial and total thyroidectomy: the first one preserves thyroid function, sparing patients the need for lifelong thyroid hormone replacement (84); moreover, microPTCs can have an excellent prognosis not requiring completion thyroidectomy. On the other hand, total thyroidectomy may present complications, such as hypoparathyroidism (often transient) (85) and recurrent laryngeal nerve injury (84), which occurs in $6 \%$ and $1 \%$ of patients, respectively (84). However, reoperation after partial thyroidectomy can be needed in cases with multifocal thyroid cancer or for radioactive iodine ablation.

In our experience, total thyroidectomy showed neither mortality nor transient and permanent nerve injuries, avoiding the risk of recurrence and necessity of completion thyroidectomy, with its known technical difficulties and increased risk of complications, and also avoiding the risk of ITC presence in remnant tissue.

Disclosure: no potential conflict of interest relevant to this article was reported.

\section{REFERENCES}

1. Bahl M, Sosa JA, Nelson RC, Esclamado RM, Choudhury KR, Hoang JK. Trends in incidentally identified thyroid cancers over a decade: a retrospective analysis of 2,090 surgical patients. World J Surg. 2014; 38:1312-17.

2. Siassakos D, Gourgiottis S, Moustafellos P, Dimopoulos N, Hadjiyannakis E. Thyroid microcarcinoma during thyroidectomy. Singapore Med J. 2008;49:23-5.

3. Trimboli P, Ulisse S, Graziano FM, Marzullo A, Ruggieri M, Calvanese $A$, et al. Trend in thyroid carcinoma size, age at diagnosis, and histology in a retrospective study of 500 cases diagnosed over 20 years. Thyroid. 2006;16:1151-5.

4. Ahn HS, Welch HG. South Korea's Thyroid-Cancer "Epidemic"-Turning theTide. N Engl J Med. 2015;373:2389-90.

5. Lin JD, Chao TC, Weng HF, Huang HS, Ho YS. Clinical presentations and treatment for 74 occult thyroid carcinoma. Comparison with nonoccult thyroid carcinoma in Taiwan. Am J Clin Oncol. 1996;19:504-8.

6. Ahmed SR, Ball DW. Clinical review: incidentally discovered 197 medullary thyroid cancer: diagnostic strategies and treatment. J Clin Endocrinol Metab. 2011;96:1237-45.

7. Boucek J, Kastner J, Skrivan J, Grosso E, Gibelli B, Giugliano $\mathrm{G}$, et al. Occult thyroid carcinoma. Acta Otorhinolaryngol Ital. 2009;29:296-304.

8. Liu H, Lv L, Yang K. Occult thyroid carcinoma: a rare case report and review of literature. Int J Clin Exp Pathol. 2014;7:5210-4.

9. Saint Marc $O$, Cogliandolo A, Piquard A, Famà F, Pidoto RR. LigaSure vs clamp-and-tie technique to achieve hemostasis in total thyroidectomy for benign multinodular goiter: a prospective randomized study. Arch Surg. 2007;142:150-6.

10. Lasithiotakis K, Grisbolaki E, Koutsomanolis D, Venianaki M, Petrakis I, Vrachassotakis N, et al. Indications for surgery and significance of unrecognized cancer in endemic multinodular goiter. World J Surg. 2012;36:1286-92.

11. Ito Y, Higashiyama T, TakamuraY, Miya A, Kobayashi K, Matsuzuka $\mathrm{F}$, et al. Prognosis of patients with benign thyroid diseases accompanied by incidental papillary carcinoma undetectable on preoperative imaging tests. World J Surg. 2007;31:1672-6.

12. Lin J, Kuo S, ChaoT, Hsueh C. Incidental and nonincidental papillary thyroid microcarcinoma. Ann Surg Oncol. 2008;15:2287-92.

13. Mangano JJ. Geographic variation in U.S. thyroid cancer incidence and a cluster near nuclear reactors in New Jersey, New York, and Pennsylvania. Int J Health Serv. 2009;39:643-61.

14. Davies L. Welch $\mathrm{H}$. Increasing incidence of thyroid cancer in the United States, 1973-2002. JAMA. 2006;295:2164-7.

15. Rizzo M, Sindoni A, Talamo Rossi R, Bonaffini O, Panetta S, Scisca $C$, et al. Annual increase in the frequency of papillary thyroid carcinoma as diagnosed by fine-needle aspiration at a cytology unit in Sicily. Hormones (Athens). 2013;12:46-57.

16. Mazzaferri EL. Managing thyroid microcarcinomas. Yonsei Med J. 2012;53:1-14.

17. Vasileiadis I, Karatzas T, Vasileiadis D, Kapetanakis S, Charitoudis G, Karakostas E, et al. Clinical and pathological characteristics of incidental and nonincidental papillary thyroid microcarcinoma in 339 patients. Head Neck. 2014;36:564-70.

18. Brito JP, AI Nofal A, Montori VM, Hay ID, Morris JC. The Impact of Subclinical Disease and Mechanism of Detection on the Rise in Thyroid Cancer Incidence: A Population-Based Study in OImsted County, Minnesota During 1935 Through 2012. Thyroid. 2015;25:999-1007.

19. Haugen BR, Alexander EK, Bible KC, Doherty GM, Mandel SJ, NikiforovYE, et al. 2015 American Thyroid Association Management Guidelines for Adult Patients with Thyroid Nodules and Differen- 
tiated Thyroid Cancer: The American Thyroid Association Guidelines Task Force on Thyroid Nodules and Differentiated Thyroid Cancer. Thyroid. 2016;26:1-133.

20. Francis GL, Waguespack SG, Bauer AJ, Angelos P, Benvenga S, Cerutti JM, et al.; American Thyroid Association Guidelines Task Force. Management Guidelines for Children with Thyroid Nodules and Differentiated Thyroid Cancer. Thyroid. 2015;25:716-59.

21. Aschebrook-Kilfoy B, Schechter RB, Shih YC, Kaplan EL, Chiu BC, Angelos $\mathrm{P}$, et al. The clinical and economic burden of a sustained increase in thyroid cancer incidence. Cancer Epidemiol Biomarkers Prev. 2013;22:1252-9.

22. Valeix P, Zarebska M, Preziosi P, Galan P, Pelletier B, Hercberg S. lodine deficiency in France. Lancet. 1999;353:1766-7.

23. Daumerie C, Ayoubi S, Rahier J, Buysschaert M, Squifflet JP. Prevalence of thyroid cancer in hot nodules. Ann Chir. 1998;52:444-8.

24. Dănilă R, Karakas E, Osei-Agyemang T, Hassan I. Outcome of incidental thyroid carcinoma in patients undergoing surgery for Graves' disease. Rev Med Chir Soc Med Nat lasi. 2008;112:115-8.

25. Pezzolla A, Marzaioli R, Lattarulo S, Docimo G, Conzo G, Ciampolillo A, et al. Incidental carcinoma of the thyroid. Int J Surg. 2014;12 Suppl 1:S98-102.

26. Pezzolla A, Lattarulo S, Milella M, Barile G, Pascazio B, Ciampolillo $A$, et al. Incidental carcinoma in thyroid pathology: our experience and review of the literature. Ann Ital Chir. 2010;81:165-9.

27. Costamagna $D$, Pagano $L$, Caputo $M$, Leutner M, Mercalli $F$, Alonzo A. Incidental cancer in patients surgically treated for benign thyroid disease. Our experience at a single institution. G Chir. 2013;34:21-6.

28. Negro R, Piana S, Ferrari M, Ragazzi M, Gardini G, Asioli S, et al. Assessing the risk of false-negative fine-needle aspiration cytology and of incidental cancer in nodular goiter. Endocr Pract. 2013;19:444-50.

29. Botrugno I, Lovisetto F, Cobianchi L, Zonta S, Klersy C, Vailati A, et al. Incidental carcinoma in multinodular goiter: risk factors. Am Surg. 2011; 77:1553-8.

30. Gelmini R, Franzoni C, Pavesi E, Cabry F, Saviano M. Incidental thyroid carcinoma (ITC): a retrospective study in a series of 737 patients treated for benign disease. Ann Ital Chir. 2010;81:421-7.

31. Pisello F, Geraci G, Sciumè C, Li Volsi F, Modica G. Total thyroidectomy of choice in papillary microcarcinoma. G Chir. 2007;28:13-9.

32. Carlini M, Giovannini C, Mercadante E, Castaldi F, Dell'Avanzato $\mathrm{R}$, Zazza S. Incidental thyroid microcarcinoma in benign thyroid disease. Incidence in a total of 100 consecutive thyroidectomies. Chir Ital. 2006;58:441-7.

33. Miccoli P, Minuto MN, Galleri D, D'Agostino J, Basolo F, Antonangeli $L$, et al. Incidental thyroid carcinoma in a large series of consecutive patients operated on for benign thyroid disease. ANZ J Surg. 2006;76:123-6.

34. Carlini M, Giovannini C, Castaldi F, Mercadante E, Dell'Avanzato R, Zazza S, et al. High risk for microcarcinoma in thyroid benign diseases. Incidence in a one year period of total thyroidectomies. J Exp Clin Cancer Res. 2005;24:231-6.

35. Pingitore R, Vignati S, Bigini D, Ciancia EM. Post-operative examination of 2930 thyroid glands: observations on primary carcinoma. Incidental carcinoma and the preoperative diagnostic assessment of thyroidectomy for cancer. Pathologica. 1993;85:591-605.

36. Pascual Corrales E, Príncipe RM, Laguna Muro S, Martínez Regueira F, Alcalde Navarrete JM, Guillén Grima F, et al. Incidental differentiated thyroid carcinoma is less prevalent in Graves' disease than in multinodular goiter. Endocrinol Nutr. 2012;59:169-73.

37. Slijepcevic N, Zivaljevic V, Marinkovic J, Sipetic S, Diklic A, Paunovic I. Retrospective evaluation of the incidental finding of 403 papillary thyroid microcarcinomas in 2466 patients undergoing thyroid surgery for presumed benign thyroid disease. BMC Cancer. 2015;15:330.
38. Zivaljević VR, Diklić AD, Krgović KLj, Zorić GV, Zivić RV, Kalezić $\mathrm{NK}$, et al. The incidence rate of thyroid microcarcinoma during surgery benign disease. Acta Chir lugosl. 2008;55:69-73.

39. Alecu L, Alecu L, Bărbulescu M, Ursuţ B, Enciu O, Slavu I, et al. Occult thyroid carcinoma in our experience - should we reconsider total thyroidectomy for benign thyroid pathology? Chirurgia (Bucur). $2014 ; 109: 191-7$.

40. Muntean V, Domsa I, Zolog A, Piciu D, Fabian O, Bosu R, et al. Incidental papillary thyroid microcarcinoma: is completion surgery required? Chirurgia (Bucur). 2013;108:490-7.

41. Lukás J, Paska J, Hintnausová B, Lukás D, Syrůcek M, Sýkorová P. The occurrence of microcarcinomas in the patients after thyroidectomy--retrospective analysis. Cas Lek Cesk. 2010;149:378-80.

42. Nechă OP, Larin OS, Cheren'ko SM, Sheptukha SA, Smoliar VA, Zolotar'ov PO. "Incidental" thyroid carcinoma among patients in surgical treatment for nontumors thyroid desease. Klin Khir. 2012;7:9-11.

43. Barczyński M, Konturek A, Stopa M, Cichoń S, Richter P, NowakW. Total thyroidectomy for benign thyroid disease: is it really worthwhile? Ann Surg. 2011;254:724-9.

44. Sakorafas GH, Stafyla V, Kolettis T, Tolumis G, Kassaras G, Peros G. Microscopic papillary thyroid cancer as an incidental finding in patients treated surgically for presumably benign thyroid disease. J Postgrad Med. 2007;53:23-6.

45. Yazici P, Mihmanli M, Bozdag E, Aygun N, Uludag M. Incidental Finding of Papillary Thyroid Carcinoma in the Patients with Primary Hyperparathyroidism. Eurasian J Med. 2015;47:194-8.

46. Tasova V, Kilicoglu B, Tuncal S, Uysal E, Sabuncuoglu MZ, Tanrikulu $Y$, et al. Evaluation of incidental thyroid cancer in patients with thyroidectomy. West Indian Med J. 2013;62:844-8.

47. Karakoyun R, Bülbüller N, Koçak S, Habibi M, Gündüz U, Erol B, et al. What do we leave behind after neartotal and subtotal thyroidectomy: just the tissue or the disease? Int J Clin Exp Med. 2013;6:922-9.

48. Berker D, Isik S, Ozuguz U, Tutuncu YA, Kucukler K, Akbaba G, et al. Prevalence of incidental thyroid cancer and its ultrasonographic features in subcentimeter thyroid nodules of patients with hyperthyroidism. Endocrine. 2011;39:13-20.

49. Tezelman S, Borucu I, Senyurek Giles Y, Tunca F, Terzioglu T. The change in surgical practice from subtotal to near-total or total thyroidectomy in the treatment of patients with benign multinodular goiter. World J Surg. 2009;33:400-5.

50. Giles Y, Boztepe H, Terzioglu T, Tezelman S. The advantage of total thyroidectomy to avoid reoperation for incidental thyroid cancer in multinodular goiter. Arch Surg. 2004;139:179-82.

51. Fernando R, Mettananda DS, Kariyakarawana L. Incidental occult carcinomas in total thyroidectomy for benign diseases of the thyroid. Ceylon Med J. 2009;54:4-6.

52. John AM, Jacob PM, Oommen R, Nair S, Nair A, Rajaratnam S. Our experience with papillary thyroid microcancer. Indian J Endocrinol Metab. 2014;18:410-3.

53. Wu Y. Occult carcinoma of the thyroid. Zhonghua Wai Ke Za Zhi. 1993;31:609-11.

54. Koh KB, Chang KW. Carcinoma in multinodular goitre. Br J Surg. 1992;79:266-7.

55. Preece J, Grodski S, Yeung M, Bailey M, Serpell J. Thyrotoxicosis does not protect against incidental papillary thyroid cancer. Surgery. 2014;156:1153-6.

56. Bron LP, O'Brien CJ. Total thyroidectomy for clinically benign disease of the thyroid gland. Br J Surg. 2004;91:569-74.

57. Bhuiyan MM, Machowski A. Nodular thyroid disease and thyroid malignancy: Experience at Polokwane Mankweng Hospital Complex, Limpopo Province, South Africa. S Afr Med J. 2015;105:570-2. 
58. Bombil I, Bentley A, Kruger D, Luvhengo TE. Incidental cancer in multinodular goitre post thyroidectomy. S Afr J Surg. 2014;52:5-9.

59. Edino ST, Mohammed AZ, Ochicha O, Malami SA, Yakubu AA. Thyroid cancers in nodular goiters in Kano, Nigeria. Niger $\mathrm{J}$ Clin Pract. 2010;13:298-300.

60. Choong KC, McHenry CR. Thyroid cancer in patients with toxic nodular goiter--is the incidence increasing? Am J Surg. 2015;209:974-6.

61. Ergin AB, Saralaya S, Olansky L. Incidental papillary thyroid carcinoma: clinical characteristics and prognostic factors among patients with Graves' disease and euthyroid goiter, Cleveland Clinic experience. Am J Otolaryngol. 2014;35:784-90.

62. Phitayakorn R, Morales-Garcia D, Wanderer J, Lubitz CC, Gaz RD, Stephen AE, et al. Surgery for Graves' disease: a 25-year perspective. Am J Surg. 2013;206:669-73.

63. Smith JJ, Chen X, Schneider DF, Broome JT, Sippel RS, Chen H, et al. Cancer after thyroidectomy: a multi-institutional experience with 1,523 patients. J Am Coll Surg. 2013;216:571-7.

64. Smith JJ, Chen X, Schneider DF, Nookala R, Broome JT, Sippel $\mathrm{RS}$, et al. Toxic nodular goiter and cancer: a compelling case for thyroidectomy. Ann Surg Oncol. 2013;20:1336-40.

65. Dunki-Jacobs E, Grannan K, McDonough S, Engel AM. Clinically unsuspected papillary microcarcinomas of the thyroid: a common finding with favourable biology? Am J Surg. 2012;203:140-4.

66. Bradly DP, Reddy V, Prinz RA, Gattuso P. Incidental papillary carcinoma in patients treated surgically for benign thyroid diseases. Surgery. 2009;146:1099-104.

67. Phitayakorn R, McHenry CR. Incidental thyroid carcinoma in patients with Graves' disease. Am J Surg. 2008;195:292-7.

68. Lokey JS, Palmer RM, Macfie JA. Unexpected findings during thyroid surgery in a regional community hospital: a 5-year experience of 738 consecutive cases. Am Surg. 2005;71:911-3.

69. Roti E, Rossi R, Trasforini G, Bertelli F, Ambrosio MR, Busutti L, et al. Clinical and histological characteristics of papillary thyroid microcarcinoma: results of a retrospective study in 243 patients. J Clin Endocrinol Metab. 2006;91:2171-8.

70. Besic N, Zgajnar J, Hocevar M, Petric R. Extent of thyroidectomy and lymphadenectomy in 254 patients with papillary thyroid microcarcinoma: a single-institution experience. Ann Surg Oncol. 2009;16:920-8.

71. Bae JS, Chae BJ, Park WC, Kim JS, Kim SH, Jung SS, et al. Incidental thyroid lesions detected by FDG-PET/CT: prevalence and risk of thyroid cancer. World J Surg Oncol. 2009;7:63.

72. Horlocker TT, Hay JE, James EM. Prevalence of incidental nodular thyroid disease detected during high-resolution parathyroid ultrasonography. In: G. Medeiros-Neto, E. Gaitan, editors. Fron- tiers in Thyroidology, Vol 2. New York: Plenum Medical; 1985. p. 1309-12.

73. Shetty SK, Maher MM, Hahn PF, Halpern EF, Aquino SL. Significance of incidental thyroid lesions detected on CT: correlation among CT, sonography, and pathology. AJR Am J Roentgenol. 2006;187:1349-56.

74. Youserm DM, Huang T, Loevner LA, Langlotz CP. Clinical and economic impact of incidental thyroid lesions found with CT and MR. AJNR Am J Neuroradiol. 1997;18:1423-8.

75. Steele SR, Martin MJ, Mullenix PS, Azarow KS, Andersen CA. The significance of incidental thyroid abnormalities identified during carotid duplex ultrasonography. Arch Surg. 2005;140:981-5.

76. Carroll BA. Asymptomatic thyroid nodules: incidental sonographic detection. AJR Am J Roentgenol . 1982;138:499-501.

77. Cohen MS, Arslan N, Dehdashti F, Doherty GM, Lairmore TC, Brunt LM, et al. Risk of malignancy in thyroid incidentalomas identified by fluorodeoxyglucose-positron emission tomography. Surgery. 2001;130:941-6.

78. Are C, Hsu JF, Schoder H, Shah JP, Larson SM, Shaha AR. FDGPET detected thyroid incidentalomas: need for further investigation? Ann Surg Oncol. 2007;14:239-47.

79. Kim TY, Kim WB, Ryu JS, Gong G, Hong SJ, Shong YK. 18F-fluoro-deoxyglucose uptake in thyroid from positron emission tomogram (PET) for evaluation in cancer patients: high prevalence of malignancy in thyroid PET incidentaloma. Laryngoscope. 2005;115:1074-8.

80. Brander A, Viikinkowski $P$, Nickels J, Kivisaari L. Thyroid gland: US screening in a random adult population. Radiology. 1991;181:683-7.

81. Ezzat S, Sarti DA, Cain DR, Braunstein GD. Thyroid incidentalomas. Prevalence by palpation and ultrasonography. Ann Intern Med. 1994;154:1838-40.

82. Fama' F, Cicciu' M, Lo Giudice G, Sindoni A, Palella J, Piquard $A$, et al. Pattern of nodal involvement in papillary thyroid cancer: a challenge of quantitative analysis. Int J Clin Exp Pathol. 2015;8:11629-34.

83. Kim J, Bang Y, Lee WJ. Living near nuclear power plants and thyroid cancer risk: A systematic review and meta-analysis. Environ Int. 2016;87:42-8.

84. Pearce EN, Braverman LE. Papillary thyroid microcarcinoma outcomes and implications for treatment. J Clin Endocrinol Metab. 2004;89:3710-2

85. Famà $F$, Cicciù $M$, Polito $F$, Cascio $A$, Gioffré-Florio $M$, Piquard $A$, et al. Parathyroid Autotransplantation During Thyroid Surgery: A Novel Technique Using a Cell Culture Nutrient Solution. World J Surg. 2016. In press. 